\title{
Optimization of PET Glycolysis Process by Response Surface Methodological Approach: A Two-Component Modelling Using Glycolysis Time and Temperature
}

\author{
Sunain Katoch, ${ }^{1}$ Vinay Sharma, ${ }^{2}$ P. P. Kundu, ${ }^{1,3}$ and M. B. Bera ${ }^{4}$ \\ ${ }^{1}$ Department of Chemical Technology, Sant Longowal Institute of Engineering \& Technology, Punjab, \\ Sangrur 148106, India \\ ${ }^{2} R M$ \& CD, Corporate Research \& Development Centre, Apollo Tyres Ltd., Limda, Gujarat, Vadodara 391760, India \\ ${ }^{3}$ Department of Polymer Science \& Technology, University of Calcutta, 92, A. P. C. Road, Kolkata 700009, India \\ ${ }^{4}$ Department of Food Engineering and Technology, Sant Longowal Institute of Engineering \& Technology, Punjab, \\ Sangrur 148106, India
}

Correspondence should be addressed to Sunain Katoch, sunainkatoch@yahoo.co.in

Received 30 January 2012; Accepted 26 February 2012

Academic Editors: A. Granville, A. Varesano, and S. Yamazaki

Copyright () 2012 Sunain Katoch et al. This is an open access article distributed under the Creative Commons Attribution License, which permits unrestricted use, distribution, and reproduction in any medium, provided the original work is properly cited.

Response surface methodology (RSM) was used for predicting the optimal condition of glycolysis time and temperature in the recycling of PET scrap. Central composite rotatable design (CCRD) for two variables at four levels was chosen as the experimental design. The data obtained from measurement of properties were fitted as a two variable second-order equation and were plotted as 3D surface plots using programme developed in MATLAB v.5. Analysis of variance (ANOVA) was used to evaluate the validity of model. The optimum operating conditions for glycolysis time and temperature were $6.5 \mathrm{~h}$ and $180^{\circ} \mathrm{C}$, respectively. Under these optimal conditions, the hydroxyl value and glycolysis conversion percentage was $38.14 \mathrm{mgKOH} / \mathrm{g}$ and $95 \%$, respectively, at the 0.97 desirability level, whereas the acid value and number average molecular weight $\left(M_{n}\right)$ at the same desirability level were $12.2 \mathrm{mgKOH} / \mathrm{g}$ and $695 \mathrm{~g} / \mathrm{mol}$, respectively.

\section{Introduction}

Polyethylene terephthalate (PET) is widely used in the manufacture of high strength fibres, soft drink bottles, and photographic films. With increasing PET consumption as packaging material, the effective utilization of PET wastes has received wide attention for the preservation of resources and protection of the environment. Since PET does not decompose readily in nature, it may be chemically depolymerized to yield original feedstock monomers. These monomers are further used for the synthesis of resins $[1,2]$. Several processes for PET depolymerization have been put forward, depending on the end use of the reclaimed products. All of these processes have both advantages and disadvantages. Hydrolysis of PET under acidic or basic conditions [3-5] gives terephthalic acid, with corrosion and pollution problems [6] and alcoholysis to dimethyl terephthalate (DMT) with liquid or gaseous methanol is possible [7]. Essentially, glycolysis involves the insertion of ethylene glycol (EG), diethylene glycol (DEG), and propylene glycol (PG) in PET chains to give bis (hydroxyethylterephthalate) (BHET) which is a substrate for PET synthesis and other oligomers.

From the alcoholysis reaction $\left(\mathrm{R}_{1} \mathrm{CO}_{2} \mathrm{R}_{2}+\mathrm{R}_{3} \mathrm{OH} \Leftrightarrow\right.$ $\mathrm{R}_{1} \mathrm{CO}_{2} \mathrm{R}_{3}+\mathrm{R}_{2} \mathrm{OH}$ ), one realizes the possibility of depolymerization of polyesters such as PET by diols (glycolysis) with a view to convert the waste into monomers for new polymer's manufacture (Scheme 1), where if $\mathrm{R}=\mathrm{C}_{2} \mathrm{H}_{4}$ then it gives BHET and if $\mathrm{R}=\mathrm{C}_{4} \mathrm{H}_{8}$ then BHBT. The reported glycolysis time varies largely. For example, a reaction time of $14 \mathrm{~h}$ is reported [8] and of only few minutes [9] in reactive extrusion.

However, a reactivity order cannot be established from results arising from different sources, because the reactivity is affected by the experimental conditions (temperature, 


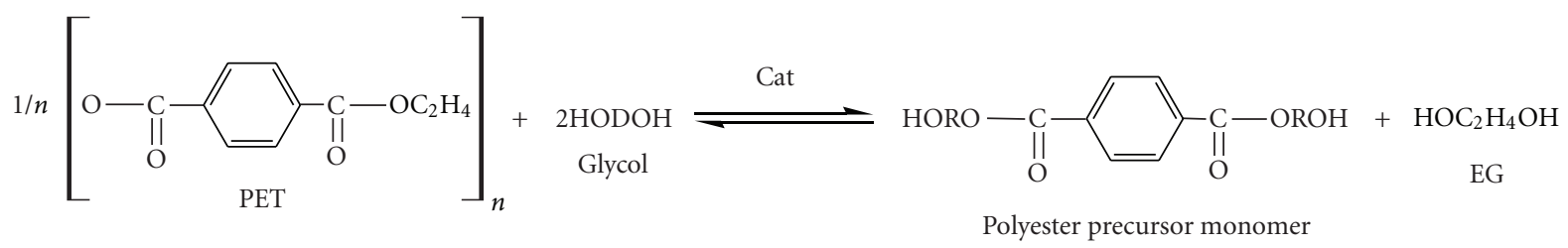

Scheme 1: Polyester precursor monomers synthesis by PET glycolysis.

TABLE 1: Two variables design matrix in the PET glycolysis process. (Coded, uncoded form of process variables).

\begin{tabular}{lcccc}
\hline S. no & $\begin{array}{c}\text { Coded values } \\
\text { Glycolysis time }\left(x_{1}\right)\end{array}$ & $\begin{array}{c}\text { Coded values } \\
\text { Glycolysis temperature }\left(x_{2}\right)\end{array}$ & $\begin{array}{c}\text { Uncoded values } \\
\text { Glycolysis time }(\mathrm{h})\left(X_{1}\right)\end{array}$ & $\begin{array}{c}\text { Uncoded values } \\
\text { Glycolysis temperature }\left({ }^{\circ} \mathrm{C}\right)\left(X_{2}\right)\end{array}$ \\
\hline 1 & -1.000 & -1.000 & 3.00 & 150.00 \\
2 & 1.000 & -1.000 & 10.00 & 150.00 \\
3 & -1.000 & 1.000 & 3.00 & 210.00 \\
4 & 1.000 & 1.000 & 10.00 & 210.00 \\
5 & -1.414 & 0.000 & 1.55 & 180.00 \\
6 & 1.414 & 0.000 & 11.45 & 180.00 \\
7 & 0.000 & -1.414 & 6.50 & 137.57 \\
8 & 0.000 & 1.414 & 6.50 & 222.43 \\
9 & 0.000 & 0.000 & 6.50 & 180.00 \\
10 & 0.000 & 0.000 & 6.50 & 180.00 \\
\hline
\end{tabular}

time, catalyst, PET/glycol ratio). Thus, little information exists on the experimental conditions that affected the glycolysis process. It was reported that reagents bearing secondary hydroxyl groups are less reactive [10]. Although PET glycolysis led to a considerable number of publications or patents, little is known about the influence of time and temperature on glycolysis process. In order to realize the influence of glycolysis temperature and glycolysis time on the depolymerization of PET flakes experiments were conducted.

In a conventional experimentation technique, experimental procedure is to hold all variables as constant while methodically changing one at a time. This technique is called one variable at a time or OVAT. The major pitfall in this type of experimental procedure is that it cannot quantitatively explore the influence of combined factor effects on the response. RSM is a collection of statistical and mathematical techniques, useful for developing, improving, and optimizing processes [11]. It also has an important application in the design, development, and formulation of new products as well as in the improvement of existing product designs. The basic components of RSM include experimental design, regression analysis, and optimization algorithms, which are used to investigate the empirical relationship between one or more measured responses and a number of independent variables, with the ultimate goal of obtaining an optimal problem solution. Based on the principle of design of experiments (DOEs), the methodology encompasses the use of various types of experimental designs, generation of polynomial equations, and mapping of the response over the experimental domain to determine the optimum formula$\operatorname{tion}(s)[12,13]$. The technique requires minimum experimentation and time, thus proving to be far more friendly and cost-effective than the conventional methods. RSM in rubber compounding was successfully used by Kundu [14] with good accuracy. If $Y$ is the response of a process dependent on the levels of $k$ factors $\left(x_{1}, x_{2}, \ldots, x_{k}\right)$, which can be precisely measured and controlled, then the model for combinations of these factor levels is given by

$$
Y_{u}=\varnothing\left(x_{1 u}, x_{2 u}, x_{3 u} \cdot x_{k u}\right)+\varepsilon_{u},
$$

where $u=1,2,3, \ldots, N=$ number of experiments; $\varnothing$ is the functional relationship between all the factors and response; and $\varepsilon$ is the error involved. Response surfaces are used in rubber science and technology in the form of ISOPLETH diagrams commonly known as contour plots. A contour plot can be defined as a series of curves that identify values of factors for which the response is constant. A response surface can be defined as a geometric representation obtained, when a response variable is plotted as a function of other variables. The sequence of operations involved in RSM are: (a) identify the variables, (b) find a suitable experimental design, (c) run experiments as dictated by design, (d) fit equations to the data, and (e) construct contours and response surfaces. Kundu and Kukreja [15] reported the coupling action of vegetable oils in rubber compounds using the conventional OVAT procedure.

The objectives of this study are to determine the optimum level of glycolysis time and temperature and their effect on various properties like acid value, hydroxyl value, $M_{n}$, and glycolysis conversion percentage. The experiments plan in coded and uncoded form of process variables is as given in Table 1. These experiments were conducted randomly. 


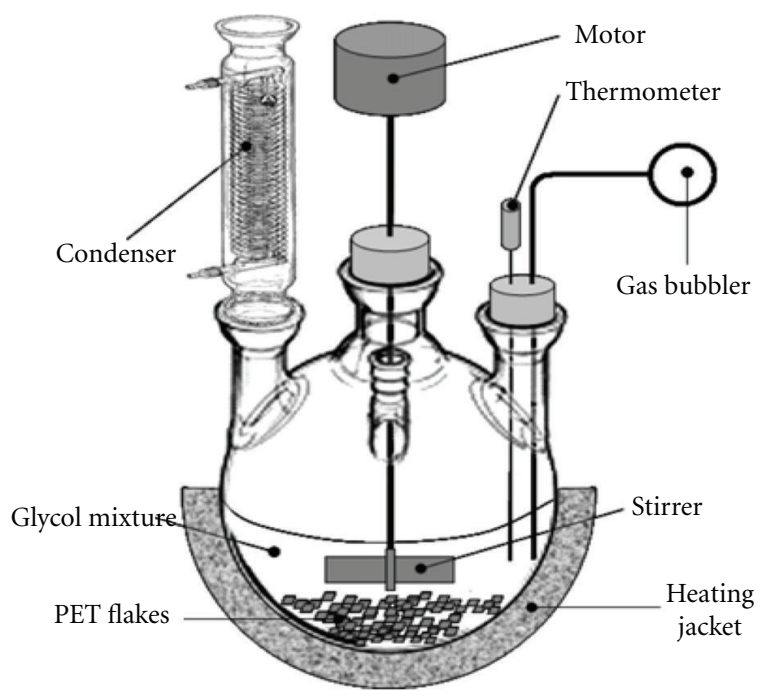

FIGURE 1: Three necked glass reactor used for glycolysis process.

\section{Experimental}

2.1. Experimental Design. CCRD is employed to fit a secondorder model. The design was generated by commercial statistical package, Design-Expert version 6.01 (Statease Inc., Minneapolis, USA, Trial version). The levels are calculated and experiments are performed using CCRD described elsewhere [16]. The two independent formulation variables selected for this particular study are the glycolysis time and temperature. The actual and corresponding coded values of different variables along with experimental data values are reported in Table 1. CCRD is an efficient and proven design, especially for two factors [16]. CCRD is also rotatable, which means that all the points in the design area are at equal distance from center. This leads to distribution of errors among all points equally. The numbers of design points in CCRD are based upon a complete $2 k$ factorial. The total numbers of experiments are

$$
N=2^{k}+2 k+m
$$

where $N$ is the total number of experiments, $k$ is the number of factors, and $m$ is the number of replicates. Multiple linear regression analysis was used, and the data was fitted as a second-order equation. The general equation that was fitted is

$$
Y=\text { constant }+a_{1} x_{1}+a_{2} x_{2}+a_{3} x_{1}^{2}+a_{4} x_{2}^{2}+a_{5} x_{1} x_{2} .
$$

The number of coefficients in the above equation is 6 . The redundancy factor of the experimental design $R_{f}=$ number of experiments/number of coefficients. For a CCRD, the numbers of experiment are 10, and the numbers of coefficients are 6. Therefore, the redundancy factor is 1.667 . The lower limits of the two variables were fixed based on the previous experimental work carried out by Mahdi et al. [17]. The choice of DEG as solvent in glycolysis is due to its more reactivity because it was reported [18] that reagents bearing secondary hydroxyl groups are less reactive.

2.2. Materials Used. Discarded PET bottles from soft drinks were procured from scrapers, cleaned thoroughly, and cut into small pieces $(6 \mathrm{~mm} \times 6 \mathrm{~mm})$. Zinc acetate, minimum assay $99 \%$, and diethylene glycol (DEG) were procured from E. Merck (India) Pvt. Ltd, Bombay. Acetic anhydride (acetylating agent) and pyridine were purchased from $\mathrm{CDH}$ (New Delhi, India) and are of analytic grade.

2.3. Glycolysis of PET Scrap. The experimental runs are formulated and ten runs were chosen from experimental design based on CCRD. The experimental region extended from -1.414 to +1.414 in terms of coded levels in independent variables of time and temperature. The lower and higher values were based on the previous research carried out by Mahdi et al. [17] and results obtained from experiments, conducted in our laboratory. Glycolysis of PET scrap has been done in a $1000 \mathrm{~mL}$ three-necked glass reactor (Figure 1) equipped with a reflux condenser, a gas bubbler (argon), a mechanical PTFE blade stirrer, and a thermometer. The whole reaction was carried out in inert atmosphere (argon atmosphere) under reflux with constant stirring.

2.4. Extraction of Water Soluble Fraction from Glycolyzed PET. After finishing each glycolysis experiment, a part of the glycolyzed product was removed from reactor, mixed with excess distilled water and the solution was vigorously agitated and filtered. The water would dissolve any unreacted glycol, catalyst, and the water soluble oligomers, while most of the glycolyzed products precipitated out. Hot water soluble oligomers (HWSOs) were extracted by boiling the glycolyzed products, after removal of excess glycol, with distilled water. The hydroxyl value, acid value, and $M_{n}$ of the 
glycolyzed product after removal of free glycol (HWSO) were determined by standard methods.

\section{Characterization}

3.1. Hydroxyl Value. The hydroxyl value was determined by following the standard method NF T 52-113.

3.2. Acid Value. To determine the acid value, sample was dissolved in a mixture of $20 \mathrm{~mL}$ of THF and $4 \mathrm{~mL}$ of water, then this solution was titrated by a solution of potassium hydroxide $(0.83 \mathrm{~N} \mathrm{KOH}$-ethanol solution). The indicator used during this titration was Bromothymol Blue. The acid value was calculated according to

$$
I_{a}=\frac{56.1\left(V_{0}-V_{1}\right) T}{m},
$$

where $V_{0}$ is the volume in millilitres of the solution of potassium hydroxide used during the blank test (without sample); $V_{1}$ is the volume in millilitres of the solution of potassium hydroxide used during the test; $m$ is the mass in grams of the sample used; $T$ is the titre value of the solution of potassium hydroxide used (in $\mathrm{mol} / \mathrm{L}$ ).

3.3. Number Average Molecular Weight. For linear polymers, the $M_{n}$ value can be calculated by determination of the end groups and using the known formula of end group analysis method [19]:

$$
M_{n}=\frac{\left(z_{m}\right)}{y}
$$

where $z$ is the number of groups that can be determined per polymer molecule, $2, m$ the mass of polymer sample, and $y$ mmol $\mathrm{KOH}$ reacted with polymer.

3.4. Glycolysis Conversion Percentage. After finishing each glycolysis experiment, the reactor was removed from the heating filament, and $200 \mathrm{~mL}$ of boiling water was slowly added into the reactor. The whole product mixture was quickly filtered using a copper mesh of $0.5 \times 0.5 \mathrm{~mm}$ pore size. The remaining depolymerized PET flakes were collected, dried, weighed, and labeled as the PET fraction. The conversion for the glycolysis of recycled PET flakes is defined as follows:

$$
\text { Glycolysis conversion percentage }=\frac{A-B}{A} \times 100,
$$

where $A$ is the weight of PET flakes before glycolysis and $B$ is the weight of PET flakes after glycolysis.

\section{Results and Discussion}

The various properties of glycolyzed product are functions of process variables (glycolysis time and temperature). In order to find out the exact quantitative relationship between response and process variables, they were fitted as a secondorder quadratic equation (3). The coefficients of the fitted equation for different properties (responses) and their percent significance in the form of $F$ ratio and $R^{2}$ (\%Fit) values are shown in Table 2. These ANOVA data were generated by using statistical tool of Microsoft Excel. The well above 76\% of $R^{2}$ value for all fitted equations indicates their insignificant level of "lack of fit;" that is, the equations are of "good fit" [20].

Figure 2 shows linear correlation plots between the actual and predicted response variables. The linear correlation plots drawn between actual and predicted responses demonstrate reasonable values of $R^{2}$; that is, for $Y_{1}$ to $Y_{4}$ it is in between 0.95 to 0.99 . The $R$-square (correlation coefficient) value provides a measure of how much of the variability in the observed response values can be explained by the experimental factors and their interactions. A good model explains most of the variations in the response.

The closer the value of $R$-square to 1.0, the stronger the model and the better are the response predictions. Hence, high values of $R$-square indicate significant lack of fit $(P<$ 0.0001 ) in both the cases. There may be other combinations of variables at which glycolysis is possible, since the primary purpose of the present study was to obtain an optimum condition at which the PET bottles are depolymerized completely and this objective was satisfactorily achieved at the previously mentioned combination of parameters; therefore other possible combinations were not tried. Tables 3(a), 3 (b) present the ANOVA demonstrating that the model is significant. The probability values, $P<0.0001$ in ANOVA, indicate significant effect of model on responses.

The process variables and responses along with their experimental values are shown in Table 4.

4.1. Hydroxyl Value. The variation of hydroxyl value with glycolysis time and temperature of the glycolyzed product is shown in Figure 3. Hydroxyl value generally shows an increasing trend with increase in glycolysis time and temperature.

It is observed that hydroxyl value increases with an increase in glycolysis time at a fixed value of glycolysis temperature. Beyond this temperature there is no further rise in hydroxyl value. The increase in hydroxyl values is probably due to hydroxyl-terminated oligomers after separation of excess glycol from GPET. Continuation of glycolysis of PET chains either by the glycol reagent or by hydroxyl-terminated oligomers leading to short chains. The first reaction leads to a consumption of $\mathrm{HODOH}$ and incorporation of $\mathrm{D}$ moieties in the chains (Scheme 1). As previously mentioned [21], glycolysis leads to a complex mixture of molecules, all of formula $\mathrm{HOZ}(\mathrm{TZ})_{n} \mathrm{OH}, n \geq 0$, which differ by their units distribution and by their degree of polymerization, $\mathrm{Z}$ and $\mathrm{T}$ being glycol and terephthalate moieties, respectively (Scheme 2). With an increase in glycolysis temperature at a fixed value of glycolysis time increase in hydroxyl value is not continuous as is evident from $3 \mathrm{D}$ surface plot. It is observed that up to a certain value of glycolysis time $(6.50 \mathrm{~h})$ the hydroxyl value shows an increasing trend, reaches a maximum, and then becomes constant. PET glycolysis never leads, as written in Scheme 1, to a pure 
TABLE 2: Coefficients of fitted equations and their percent significance $Y=a_{0}+a_{1} x_{1}+a_{2} x_{2}+a_{3} x_{1} x_{2}+a_{4} x_{1}^{2}+a_{5} x_{2}^{2}$.

\begin{tabular}{lccccccccc}
\hline S. no. & Property & $a_{0}$ & $a_{1}$ & $a_{2}$ & $a_{3}$ & $a_{4}$ & $a_{5}$ & $F$-ratio & $\%$ Fit \\
\hline 1 & Hydroxyl value & 37.54 & 2.58 & 1.36 & -0.30 & -1.96 & -0.50 & 8.96 & 86.4 \\
2 & Acid value & 15.40 & -0.43 & -0.11 & -0.65 & -0.47 & -1.42 & 38.52 & 96.4 \\
3 & Av. mol. wt & 700.00 & -181.76 & 139.52 & 66.50 & 171.31 & 76.56 & 8.04 & 85.1 \\
4 & Glycolysis conversion \% & 95.00 & 28.15 & 12.53 & 11.25 & -19.50 & 25.25 & 342.88 & 99.5 \\
\hline
\end{tabular}

TABLE 3: (a) Summary of results in analyzing lack of fit (LOF) for hydroxyl and acid value. (b) Summary of results in analyzing lack of fit (LOF) for number average molecular weight and glycolysis conversion percentage.

(a)

\begin{tabular}{|c|c|c|c|c|c|}
\hline \multicolumn{6}{|c|}{ ANOVA for response surface quadratic model (hydroxyl value) } \\
\hline Source & Sum of squares & DF & Mean square & $F$ value & $P$ \\
\hline Model & 95.57 & 5 & 19.11 & 8.96 & $<0.0001$ \\
\hline Residual & 14.93 & 7 & 2.13 & & \\
\hline Lack of fit & 7.63 & 3 & 2.54 & 1.19 & \\
\hline Pure error & 7.30 & 4 & 1.82 & & \\
\hline \multicolumn{6}{|c|}{ ANOVA for response surface quadratic model (acid value) } \\
\hline Model & 17.97 & 5 & 3.59 & 38.52 & $<0.0001$ \\
\hline Residual & 0.65 & 7 & 0.093 & & \\
\hline Lack of fit & 0.65 & 3 & 0.22 & 2.365 & \\
\hline Pure error & 0.000 & 4 & 0.000 & & \\
\hline
\end{tabular}

(b)

\begin{tabular}{lccccc}
\hline & \multicolumn{5}{c}{ ANOVA for response surface quadratic model $\left(M_{n}\right)$} \\
Source & Sum of squares & DF & Mean square & $F$ value & 8.04 \\
\hline Model & 6.627 & 5 & 1.325 & \\
Residual & 1.153 & 7 & 16476.43 & 2.33 \\
Lack of fit & 1.153 & 3 & 38445.01 & \\
Pure error & 0.000 & 4 & 0.000 & $<0001$ \\
\hline & ANOVA for response surface quadratic model (glycolysis conversion percentage) & \\
Model & 14396.45 & 5 & 2879.29 & 2.0001 \\
Residual & 58.78 & 7 & 8.40 & \\
Lack of fit & 58.78 & 3 & 19.59 & \\
Pure error & & 4 & &
\end{tabular}

TABLE 4: Experimental values of responses and variables for glycolysis process.

\begin{tabular}{|c|c|c|c|c|c|}
\hline $\begin{array}{l}\text { Glycolysis } \\
\text { Time }(\mathrm{h})\left(X_{1}\right)\end{array}$ & $\begin{array}{c}\text { Glycolysis } \\
\text { Temperature }\left({ }^{\circ} \mathrm{C}\right)\left(X_{2}\right)\end{array}$ & $\begin{array}{c}\text { Hydroxyl } \\
\text { values }\left(Y_{1}\right)\end{array}$ & $\begin{array}{l}\text { Acid values } \\
\qquad\left(Y_{2}\right)\end{array}$ & $\begin{array}{c}\text { Average molecular } \\
\text { Wt. }\left(Y_{3}\right)\end{array}$ & $\begin{array}{c}\text { Conversion } \\
\text { percentage }\left(Y_{4}\right)\end{array}$ \\
\hline 3.00 & 150.00 & 32.16 & 12.8 & 1500 & 20 \\
\hline 10.00 & 150.00 & 36.12 & 13.7 & 1014 & 50 \\
\hline 3.00 & 210.00 & 35.38 & 12.9 & 915 & 25 \\
\hline 10.00 & 210.00 & 38.14 & 12.4 & 700 & 95 \\
\hline 1.55 & 180.00 & 28.31 & 12.7 & 1224 & 15 \\
\hline 11.45 & 180.00 & 38.14 & 13.1 & 695 & 95 \\
\hline 6.50 & 137.57 & 34.16 & 12.9 & 845 & 30 \\
\hline 6.50 & 222.43 & 38.14 & 15.4 & 700 & 62 \\
\hline 6.50 & 180.00 & 38.14 & 12.2 & 695 & 95 \\
\hline 6.50 & 180.00 & 38.14 & 12.2 & 695 & 95 \\
\hline
\end{tabular}




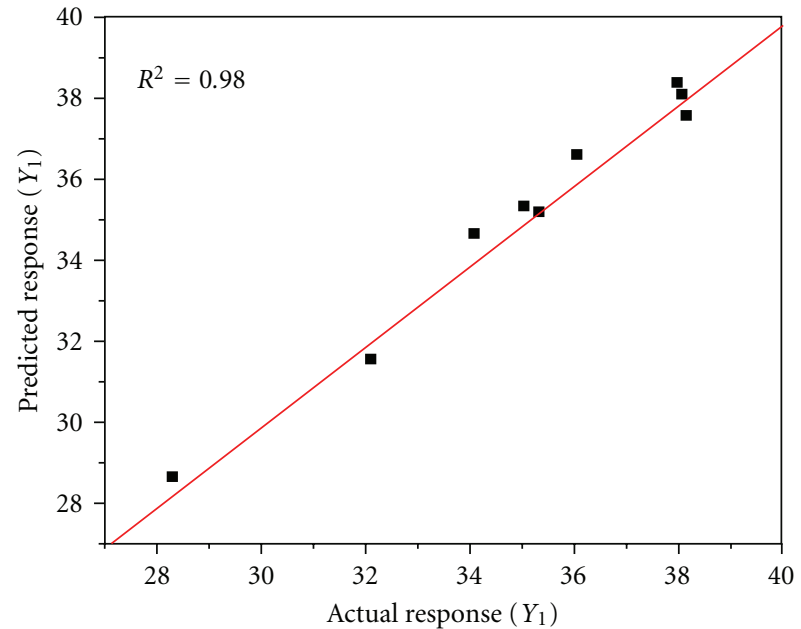

(a)

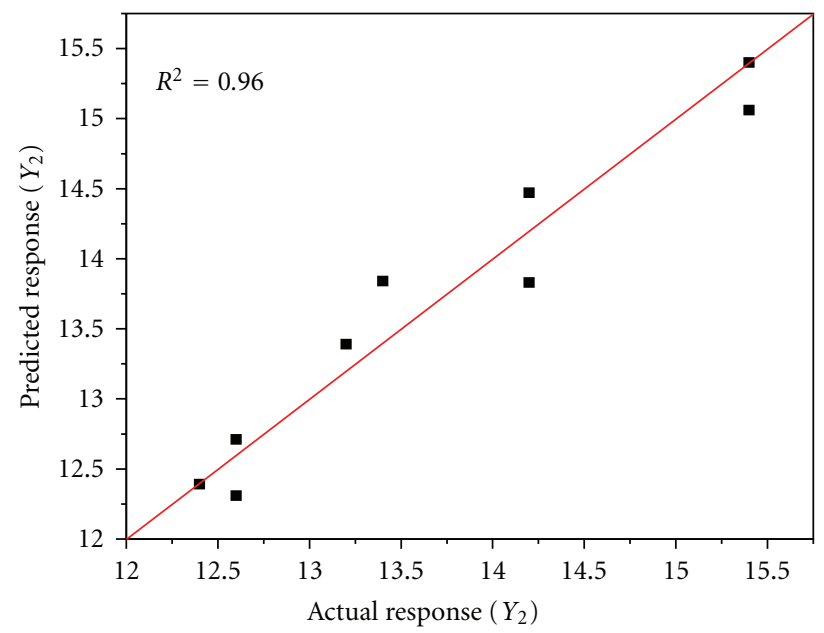

(c)

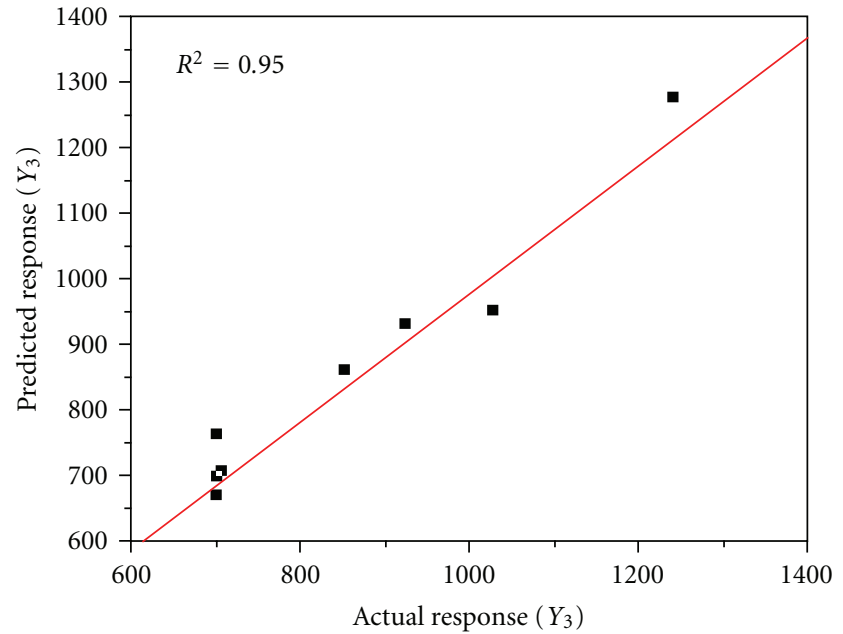

(b)

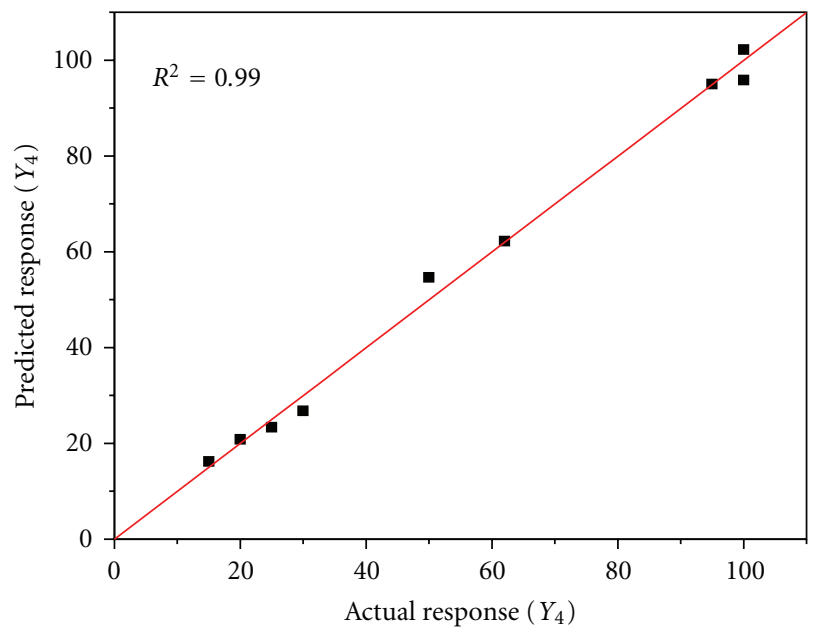

(d)

FIGURE 2: Linear correlation plots between actual and predicted respon. (a) hydroxyl value, (b) acid value, (c) $M_{n}$, and (d) glycolysis conversion percentage, respectively.

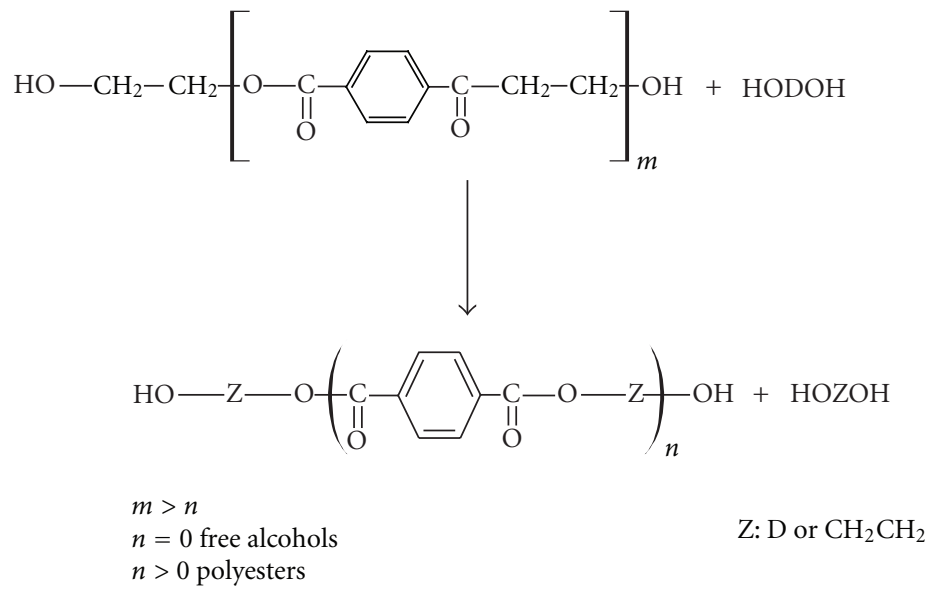

$1 / n(\mathrm{ET})_{n}+\mathrm{HODOH} \longrightarrow$ stat $\mathrm{HO}(\mathrm{E})_{k}(\mathrm{D})_{l}(\mathrm{TE})_{m}(\mathrm{TD})_{p} \mathrm{OH}$ symbolized as $\mathrm{HOZ}(\mathrm{TZ})_{n} \mathrm{OH}$

Scheme 2: Glycolysis of PET (written with a simplified notation). 


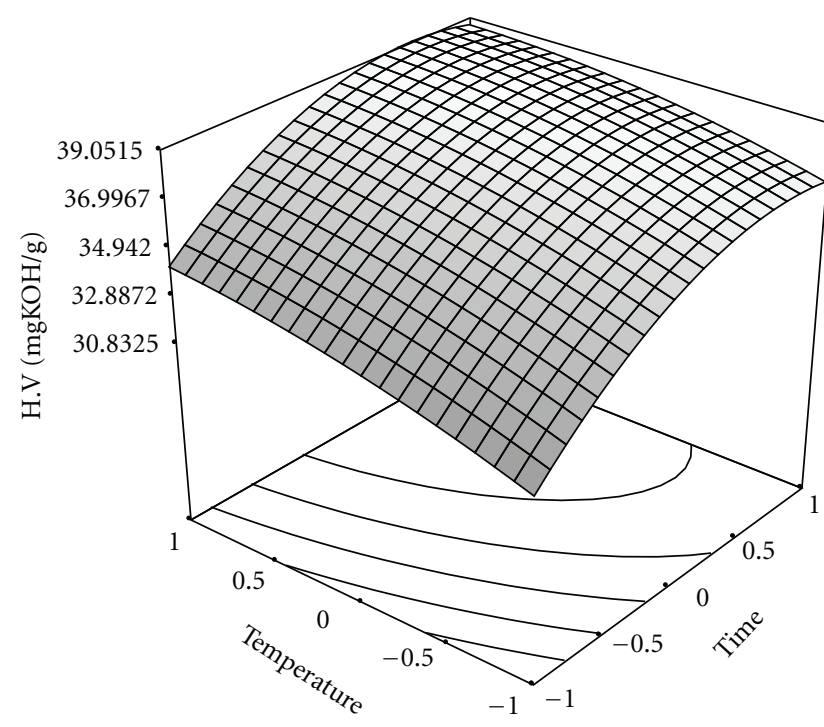

Figure 3: Surface plot (3D) showing variation in hydroxyl value with glycolysis time and temperature.

dihydroxyalkyl terephthalate. The cause is the multiplicity of reversible glycolysis reactions (between all esters and all glycol species). To simplify, one may separate them into three classes: "interchange," "polycondensation," and "glycolysis" reactions. It is concluded that beyond the optimum level of glycolysis time (around $6.50 \mathrm{~h}$ ) at a fixed temperature $\left(180^{\circ} \mathrm{C}\right)$, there is no change in the hydroxyl value of glycolyzed product. This indicates that the equilibrium has been established due to polycondensation reaction. The equation of the model fitted for hydroxyl value in the actual form of process variables is

$$
\begin{aligned}
\text { Hydroxyl value }\left(\frac{\mathrm{mgKOH}}{\mathrm{g}}\right) & \\
= & -3.60+3.33 \times X_{1}+0.26 \times X_{2}-0.16 \times\left(X_{1}\right)^{2} \\
& -5.58 \times\left(X_{2}\right)^{2}-2.85 \times X_{1} \times X_{2} .
\end{aligned}
$$

4.2. Acid Value. The change in acid value with glycolysis time and temperature is plotted as $3 \mathrm{D}$ surface plot is shown in Figure 4. There is a general perception that acid value is greatly affected by glycolysis time and temperature due to sidewise hydrolysis reaction that leads to certain fraction of terephthalate oligoester. With increase in glycolysis temperature at a fixed glycolysis time, the increase in hydroxyl value is not continuous as evident from contour plots. It is observed that, up to a certain value of glycolysis time $(6.50 \mathrm{~h})$, the hydroxyl value shows an increasing trend, reaches a maximum, and then starts decreasing. Although the glycolysis of PET should yield oligomers with hydroxyl end groups, but the acid value indicates that insignificant hydrolysis may be occurring during glycolysis reaction, due to presence of water as impurity or formed by side reactions, and some products with carboxyl end groups are also formed, especially at lower amounts of DEG.

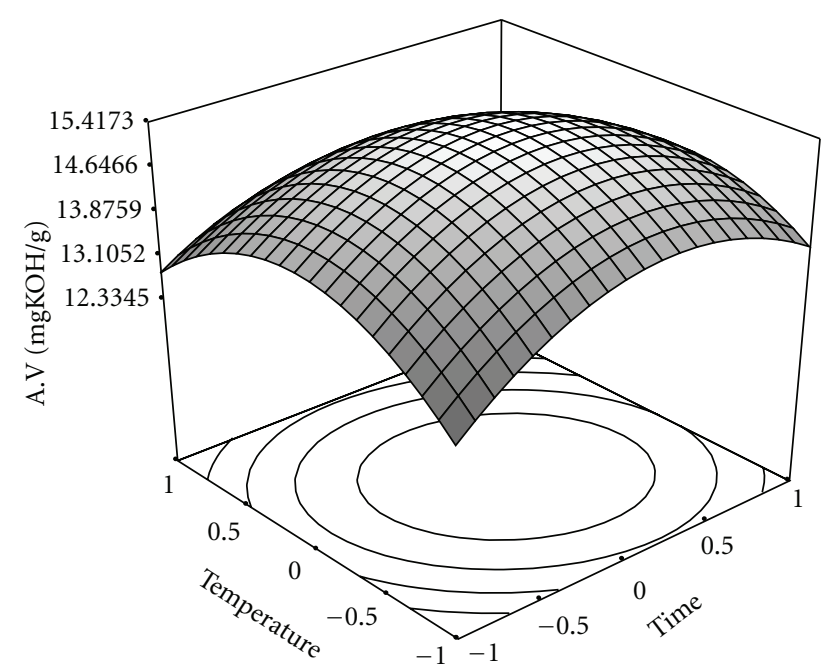

Figure 4: Surface plot (3D) showing variation in acid value with glycolysis time and temperature.

Such hydrolysis reaction has also been observed by Cüçlü et al. [22]. According to Yoon et al. [23], the etherification reactions yield polymers with DEG end groups and water formation or resulting formation of polymers with hydroxylcarboxyl and carboxylic end groups. The evolved water can react further increasing the fraction of carboxylic end group products, EG monomers and continuing the etherification reactions. It was found, that with an increase in the glycolysis time at fixed temperature, the glycolyzed product after removal of unreacted glycol had higher acid value. Beyond this temperature, there is fall in hydroxyl value. It might be due to retardation in the growth of side reaction at higher temperature which leads to the hydrolysis reaction. Hence, the production of minor fractions of terephthalate oligoester (carboxyl terminated oligomers) is minimized. This leads to decrease in acid value. The equation of the model fitted for acid value in the actual form of process variables is:

$$
\begin{aligned}
\text { Acid value }\left(\frac{\mathrm{mgKOH}}{\mathrm{g}}\right) & \begin{aligned}
= & -40.47+1.90 \times X_{1}+0.56 \times X_{2}-0.09 \times\left(X_{1}\right)^{2} \\
& -1.52 \times\left(X_{2}\right)^{2}-3.33 \times X_{1} \times X_{2} .
\end{aligned}
\end{aligned}
$$

4.3. Number Average Molecular Weight. The glycolysis shows the reduction of recycled PET to lower molecular weight fragments. The variation of $M_{n}$ of the glycolyzed product with the glycolysis time and temperature is shown in Figure 5 as $3 \mathrm{D}$ surface plot. With increase in glycolysis time and temperature, $M_{n}$ generally shows decreasing trend.

Contour plot indicates that initially, at low time and temperature, $M_{n}$ was highest. The longer reaction leads to further decrease in molecular weight, but after seven hours, the decrease in molecular weight is quite moderate. The $M_{n}$ decreases with an increase in glycolysis time at a fixed glycolysis temperature. There is no change observed in value 


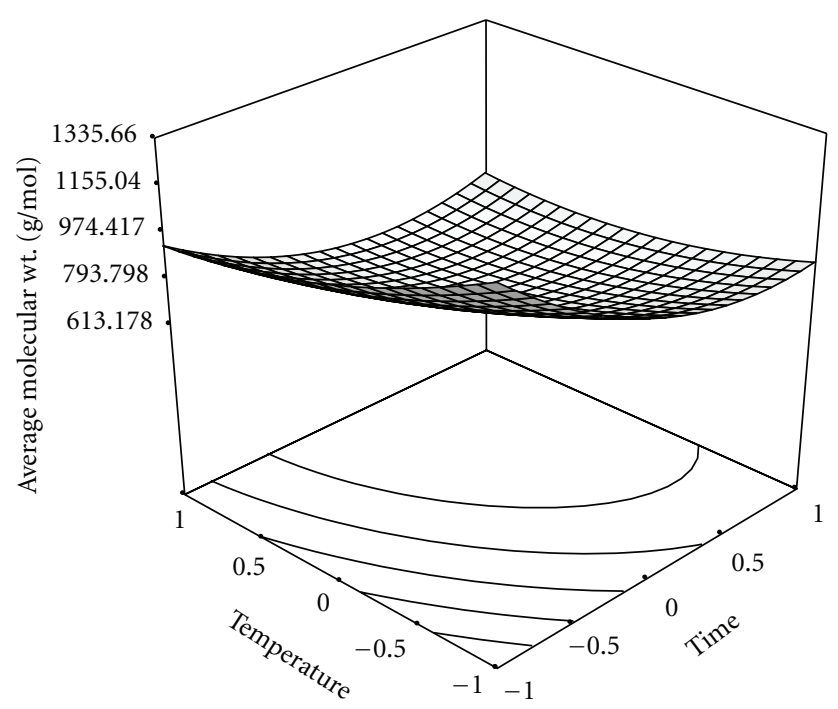

FIGURE 5: Surface plot (3D) showing variation in number average molecular weight with glycolysis time and temperature.

beyond that temperature. With increase in glycolysis temperature at a fixed glycolysis time, the decrease in $M_{n}$ value is not continuous as is evident from surface plot. It is observed that, up to a certain value of glycolysis time $(6.50 \mathrm{~h})$, the $M_{n}$ shows the decreasing trend and reaches a minimum and then becomes constant. These results were explained by a mechanism of chains scission at the amorphous interlamellar segments by the diffused DEG, crack formation by the internal tensions created and final mechanical disintegration of the scraps. After this initial period, glycolysis reactions continue in the liquid phase until the equilibrium state is reached.

The equation of the model fitted for $M_{n}$ in the actual form of process variables is

$$
\begin{aligned}
M_{n}= & +5962.76-347.73 \times X_{1}-39.39 \times X_{2} \\
& +13.98 \times\left(X_{1}\right)^{2}+0.08 \times\left(X_{2}\right)^{2}+0.63 \times X_{1} \times X_{2} .
\end{aligned}
$$

4.4. Glycolysis Conversion Percentage. The variation of glycolysis conversion percentage with glycolysis time and temperature of the glycolyzed product is shown in Figure 6 as 3D surface plot. With increase in glycolysis time and temperature glycolysis conversion percentage generally shows increasing trend. It was observed that the glycolysis conversion increased with an increase in the glycolysis time at fixed temperature. It approached a steady state at about $6.50 \mathrm{~h}$ (95\% conversion).

With increase in glycolysis temperature at a fixed value of glycolysis time increase in glycolysis conversion percentage is not increasing continuously as is evident from surface plot. It is observed that up to a certain value of glycolysis time $(6.50 \mathrm{~h})$ the glycolysis conversion percentage shows an increasing trend, reaches a maximum, and then attains steady state. It was found that the glycolysis conversion was 30\% at

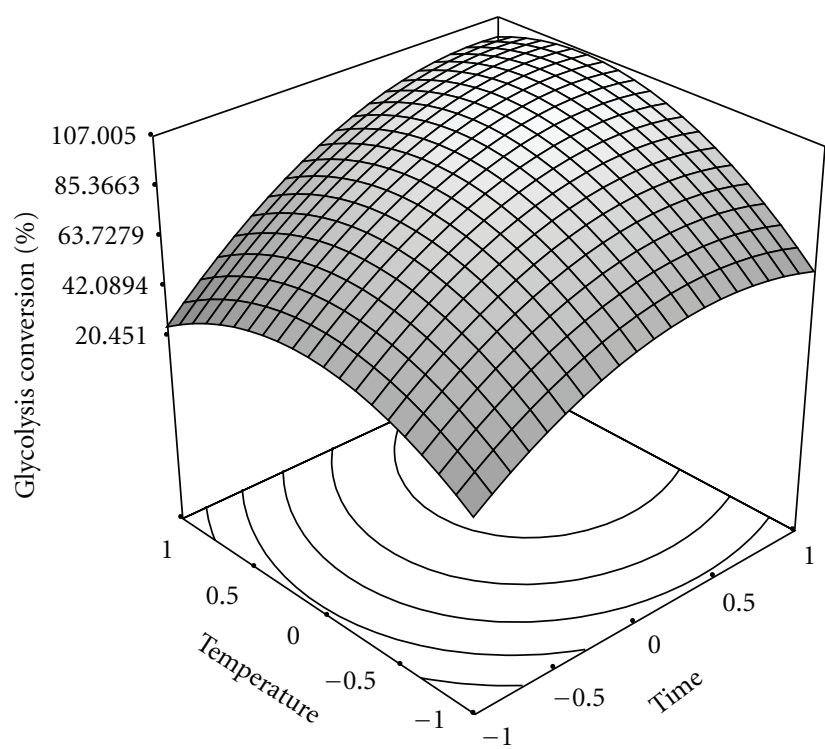

FIGURE 6: Surface plot (2D) showing variation in Glycolysis conversion percentage with glycolysis time and temperature.

glycolysis temperature below $140^{\circ} \mathrm{C}$. The glycolysis conversion slowly increased after $140^{\circ} \mathrm{C}$ and was $50 \%$ at $150^{\circ} \mathrm{C}$ and even $95 \%$ beyond $180^{\circ} \mathrm{C}$. At lower temperature (e.g., below $140^{\circ} \mathrm{C}$ ) the reaction is very slow but the composition of the liquid phase seems to be always near the thermodynamic equilibrium state corresponding to the local stoichiometric ratio of glycolic to terephthalic units. This could result from a slow release of partially depolymerised polyesters from the solid phase to the liquid phase, leaving them the time to be glycolyzed in the liquid phase nearly until equilibrium state. Increasing the temperature above $180^{\circ} \mathrm{C}$ leads to a high acceleration of release in liquid phase of partially depolymerized polyesters that have not been glycolyzed until equilibrium state.

The equation of the model fitted for glycolysis conversion percentage in the actual form of process variables is

$$
\begin{aligned}
& \text { Glycolysis conversion percentage } \\
& \qquad \begin{aligned}
= & -895.05+12.48 \times X_{1}+9.87 \times X_{2} \\
& -1.69 \times\left(X_{1}\right)^{2}-0.02 \times\left(X_{2}\right)^{2}+0.09 \times X_{1} \times X_{2} .
\end{aligned}
\end{aligned}
$$

4.5. Optimization of Glycolysis Process. In order to optimize the process conditions for the glycolysis process by numerical optimization technique, the main aim was to get maximum hydroxyl value and glycolysis conversion percentage. Also the minimum acid value and $M_{n}$ confirmed the extent of depolymerization. The optimum operating conditions for glycolysis time and temperature were $6.50 \mathrm{~h}$ and $180^{\circ} \mathrm{C}$. Under these optimal conditions, the hydroxyl value and glycolysis conversion percentage were found to be $38.14 \mathrm{mgKOH} / \mathrm{g}$ and $95 \%$, respectively, at the 0.98 desirability level, whereas the acid 
value and $M_{n}$ at the same desirability level was observed to be $12.22 \mathrm{mgKOH} / \mathrm{g}$ and 695 , respectively.

\section{Conclusion}

The effect of different process variables for the glycolysis of PET scrap like glycolysis time and temperature on hydroxyl value, acid value, $M_{n}$ and glycolysis conversion percentage has been studied and analyzed. Response surface methodology (RSM) using central composite rotatable design (CCRD) was employed to analyze and optimize the glycolysis process. Analysis of variance (ANOVA) was also used to evaluate the validity of the model. The optimum operating conditions for glycolysis time and temperature were $6.50 \mathrm{~h}$ and $180^{\circ} \mathrm{C}$. Under these optimal conditions, the hydroxyl value and glycolysis conversion percentage was found to be $38.14 \mathrm{mgKOH} / \mathrm{g}$ and $95 \%$, respectively, at the 0.97 desirability level, whereas the acid value and $M_{n}$ at the same desirability level were observed to be $12.22 \mathrm{mgKOH} / \mathrm{g}$ and $695 \mathrm{~g} /$ mol, respectively. The statistical parameters reveal strong evidence that the constructed models for variables are reliable.

\section{Acknowledgments}

S. Katoch is highly thankful to Dr. Bahadur Singh and Dr. Kamlesh Prasad of Deptartment of Food Engineering and Technology, SLIET, Longowal, Punjab, India for help in response surface methodology and analysis of variance.

\section{References}

[1] D. Paszun and T. Spychaj, "Chemical recycling of poly(ethylene terephthalate)," Industrial and Engineering Chemistry Research, vol. 36, no. 4, pp. 1373-1383, 1997.

[2] U. R. Vaidya and V. M. Nadkarni, "Polyester polyols for polyurethanes from pet waste. Kinetics of polycondensation," Journal of Applied Polymer Science, vol. 35, no. 3, pp. 775-785, 1988.

[3] J. R. Campanelli, M. R. Kamal, and D. G. Cooper, "Kinetic study of the hydrolytic degradation of polyethylene terephthalate at high temperatures," Journal of Applied Polymer Science, vol. 48, no. 3, pp. 443-451, 1993.

[4] J. R. Campanelli, D. G. Cooper, and M. R. Kamal, "Catalyzed hydrolysis of polyethylene terephthalate melts," Journal of Applied Polymer Science, vol. 53, no. 8, pp. 985-991, 1994.

[5] G. P. Karayannidis, A. P. Chatziavgoustis, and D. S. Achilias, "Poly(ethylene terephthalate) recycling and recovery of pure terephthalic acid by alkaline hydrolysis," Advances in Polymer Technology, vol. 21, no. 4, pp. 250-259, 2002.

[6] S. Baliga and W. T. Wong, "Depolymerization of poly(ethylene terephthalate) recycled from post-consumer soft-drink bottles," Journal of Polymer Science Part A, vol. 27, no. 6, pp. 20712082, 1989.

[7] J. Y. Chen, C. F. Ou, Y. C. Hu, and C. C. Lin, "Depolymerization of poly (ethylene terephthalate) resin under pressure," Journal of Applied Polymer Science, vol. 42, no. 6, pp. 15011507, 1991.

[8] D. A. Heyman and O. M. Grace, "High polymer content graft polyols," Journal of Cellular Plastics, vol. 21, no. 2, pp. 101-104, 1985.
[9] M. Dannoux, P. Cassagnau, and A. Michel, "Synthesis of oligoester $\alpha, \omega$-diols by alcoholysis of PET through the reactive extrusion process," Canadian Journal of Chemical Engineering, vol. 80, no. 6, pp. 1075-1082, 2002.

[10] J. S. Canaday and M. J. Skowronski, "Comparison of aromatic polyester polyols for rigid urethane and isocyanurate foam," Journal of Cellular Plastics, vol. 21, no. 5, pp. 338-344, 1985.

[11] R. H. Myers and D. C. Montgomery, Response Surface Methodology: Process and Product Optimization Using Designed Experiments, Wiley, New York, NY, USA, 1995.

[12] M. Kincl, S. Turk, and F. Vrečer, "Application of experimental design methodology in development and optimization of drug release method," International Journal of Pharmaceutics, vol. 291, no. 1-2, pp. 39-49, 2005.

[13] T. R. Kukreja, D. Kumar, K. Prasad, R. C. Chauhan, S. Choe, and P. P. Kundu, "Optimisation of physical and mechanical properties of rubber compounds by response surface methodology-two component modelling using vegetable oil and carbon black," European Polymer Journal, vol. 38, no. 7, pp. 1417-1422, 2002.

[14] P. P. Kundu, "Improvement of filler-rubber interaction by the coupling action of vegetable oil in carbon black reinforced rubber," Journal of Applied Polymer Science, vol. 75, no. 6, pp. 735-739, 2000.

[15] P. P. Kundu and T. R. Kukreja, "Surface modification of carbon black by vegetable oil-its effect on the rheometric, hardness, abrasion, rebound resilience, tensile, tear, and adhesion properties," Journal of Applied Polymer Science, vol. 84, no. 2, pp. 256-260, 2002.

[16] C. F. Jeff Wu and M. Hamada, Experiments Planning, Analysis, and Parameter Design Optimization, Wiley, New York, NY, USA, 2000.

[17] F. Mahdi, A. A. Khan, and H. Abbas, "Physiochemical properties of polymer mortar composites using resins derived from post-consumer PET bottles," Cement and Concrete Composites, vol. 29, no. 3, pp. 241-248, 2007.

[18] G. Tersac, F. Hubert, G. Durand, J. Quillerou, J. Rivéro, and J. C. Maréchal, "Polyols by polyestérification of glycolysis products of polyethyleneterephthalate, Their Physicochemical Properties and Resultant Polyurethane Foams," Cellular Polymers, vol. 14, no. 1, pp. 14-40, 1995.

[19] B. H. Stuart, Polymer Analysis, vol. 116, chapter 4, John Wiley \& Sons, New York, NY, USA, 2002.

[20] D. C. Montgomery, Design and Analysis of Experiments, vol. 229, chapters 6 and 7, John Wiley \& Sons, New York, NY, USA, 1991.

[21] F. Hubert, G. Durand, and G. Tersac, "Equilibria in the alcoholysis reactions of terephthalic esters and chemical valorization of polyethyleneterephthalate waste-I. Equilibrium constants determination," Journal of Applied Polymer Science, vol. 72, no. 3, pp. 329-340, 1999.

[22] G. Cüçlü, A. Kaşgöz, S. Özbudak, S. Özgümüş, and M. Orbay, "Glycolysis of poly(ethylene terephthalate) wastes in xylene," Journal of Applied Polymer Science, vol. 69, no. 12, pp. 23112319, 1998.

[23] K. H. Yoon, M. H. Kwon, M. H. Jeon, and O. O. Park, "Diffusion of ethylene glycol in solid state poly(ethylene terephthalate)," Polymer Journal, vol. 25, no. 3, pp. 219-226, 1993. 

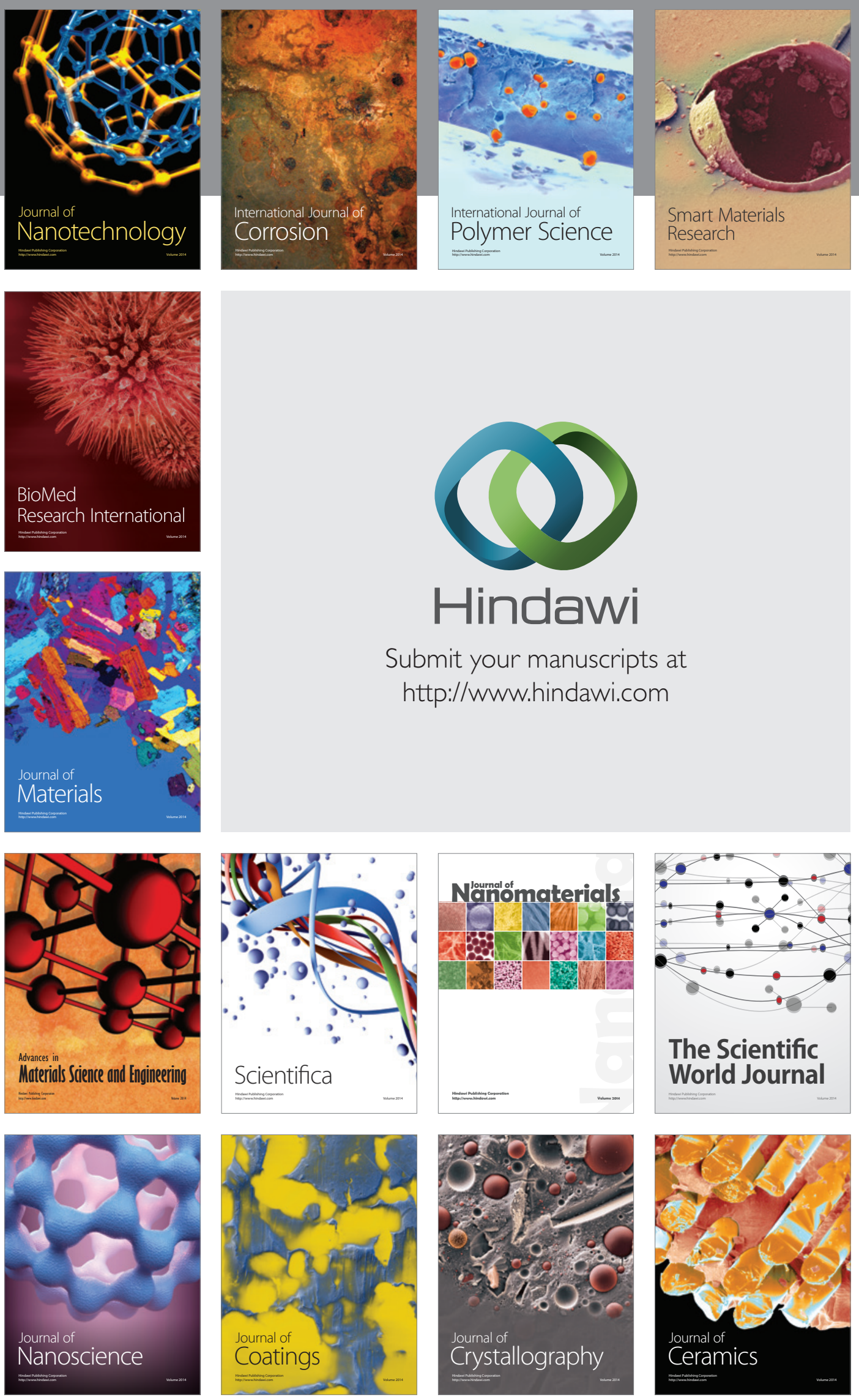

The Scientific World Journal

Submit your manuscripts at

http://www.hindawi.com

\section{World Journal}

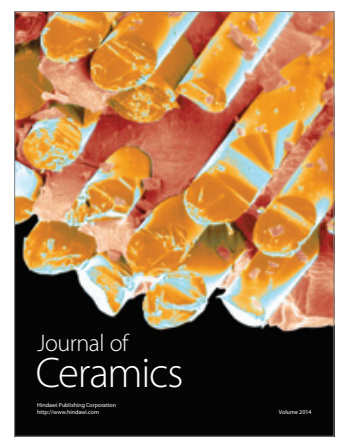

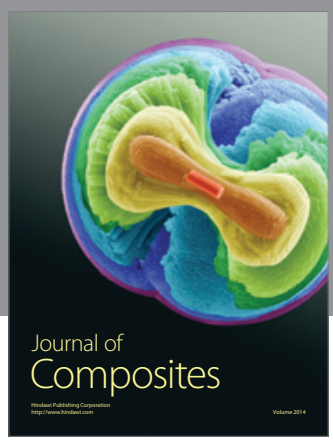
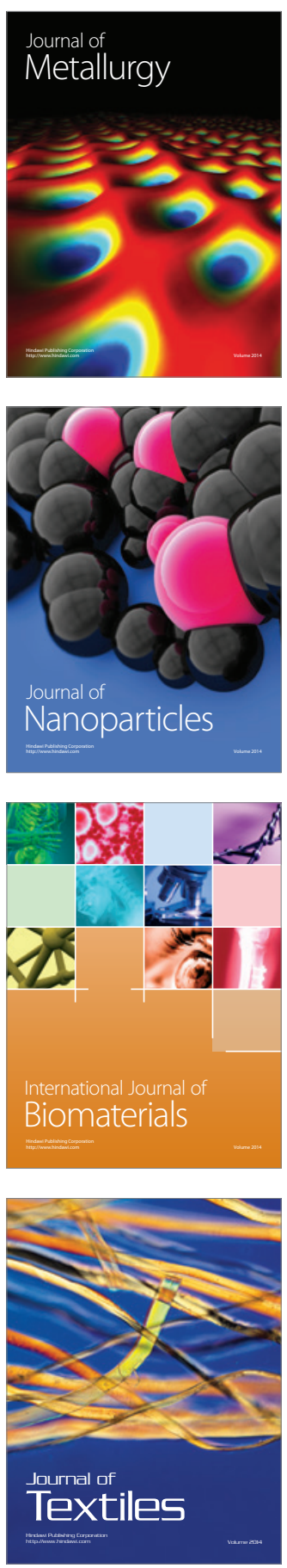